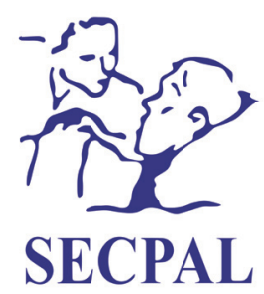

Medicina Paliativa

www.medicinapaliativa.es

ORIGINAL

\title{
Miedo a la muerte y su relación con la inteligencia emocional en estudiantes de enfermería
}

\author{
Elena Fernández-Martínez"1 ${ }^{1}$ Cristina Liébana-Presa*1, María Martínez-Soriano ${ }^{2}$ \\ y Ana López-Alonso ${ }^{3}$
}

${ }^{1}$ Grupo de investigación SALBIS, Facultad de Ciencias de la Salud, Universidad de León, León, España. ${ }^{2}$ Hospital Comarcal de Alcañiz, Teruel, España. ${ }^{3}$ Grupo de Investigación Trasplante, Facultad de Ciencias de la Salud, Universidad de León, León, España

Recibido el 18 de junio de 2018

Aceptado el 28 de julio de 2018

\author{
PALABRAS CLAVE \\ Inteligencia \\ emocional, \\ emociones, miedo a la \\ muerte, muerte, \\ estudiantes de \\ enfermería.
}

\begin{abstract}
Resumen
Antecedentes y objetivo: Los estudiantes de enfermería deben adquirir competencias emocionales para afrontar la muerte como parte de su etapa formativa. Con este estudio se plantea describir las variables miedo a la muerte e inteligencia emocional, analizar la relación entre ellas y comparar si existen diferencias con otras variables sociodemográficas.

Método: Estudio descriptivo transversal, diseño comparativo y correlacional con una población de 164 estudiantes de cuarto de Grado en Enfermería. La recogida de datos fue un cuestionario anónimo que contenía variables sociodemográficas, la escala Miedo a la Muerte de Collett-Lester (EMMCL) y la escala de inteligencia emocional TMMS-24. Se analizaron estadísticos descriptivos, coeficiente de correlación de Pearson y se aplicó la prueba de T de Student.

Resultados: Participaron 101 estudiantes (61,6 \%). Se obtuvo una correlación inversa entre la regulación emocional y el miedo a la muerte. Hay diferencias significativas entre el miedo a la muerte y el género. Las experiencias previas relacionadas con la muerte y la preparación en el cuidado del enfermo terminal se correlacionan inversamente con el miedo a la muerte.

Conclusiones: Las mujeres alcanzan mayores puntuaciones de miedo a la muerte que los hombres. Además, a mayor regulación de las emociones, menor miedo a la muerte. Es importante conocer las actitudes ante la muerte e implementar programas de formación en habilidades emocionales entre los estudiantes de enfermería.
\end{abstract} \#Articulo extraído del Trabajo Fin de Máster titulado: “Miedo a la muerte y su relación con la inteligencia emocional en estudiantes de
enfermería” presentado en el Máster Universitario de Investigación en Ciencias Socio-sanitarias de la Universidad de León (España).

*Autor para correspondencia:

Cristina Liébana-Presa

Facultad de Ciencias de la Salud, Universidad de León, Campus de Ponferrada. Avda. Astorga s/n. 24401 Ponferrada, León, España

Correo electrónico: cliep@unileon.es 
KEYWORDS

Emotional

intelligence, emotions, fear of death, death, nursing students.

\begin{abstract}
Background and objectives: Nursing students must build emotional competence for facing death as part of their training program. This study was performed to describe the variables fear of death and emotional intelligence, to analyze the relationship between them, and to assess whether any differences exist with other sociodemographic variables.

Method: This was a comparative, correlational, cross-sectional study in a population of 164 fourthyear nursing degree students. Data were collected using an anonymous questionnaire, which included sociodemographic variables, the Collett-Lester Fear of Death Scale (CL-FODS or EMMCL in Spanish), and the Trait Meta-Mood Scale 24 (TMMS-24) for emotional intelligence. Descriptive statistics and Pearson's correlation coefficient were analyzed; Student's t-test was also used.

Results: A total of 101 students took part in the study (61.6\%). An inverse correlation was obtained between emotional regulation and fear of death. Significant differences exist between fear of death and gender. Prior experiences related to death and terminal care training are inversely correlated with fear of death.

Conclusions: Women obtained higher fear of death scores when compared to men. Furthermore, the greater the emotional regulation, the lower the fear of death. It is important that attitudes towards death be acknowledged, and that training programs in emotional skills be provided to nursing students.
\end{abstract}

Fernández-Martínez E, Liébana-Presa C, Martínez-Soriano M, López-Alonso A. Miedo a la muerte y su relación con la inteligencia emocional en estudiantes de enfermería. Med Paliat. 2019;26(3):205-210.

\section{Introducción}

La muerte, además de ser un hecho biológico, cuenta con una dimensión psicológica, social y cultural que varía según el momento histórico, las costumbres, creencias y códigos de la sociedad donde acontece ${ }^{1}$. La convivencia con la muerte también ha pasado de ser un acto íntimo que tenía lugar en el entorno familiar a ser un acto que tiene lugar en una institución hospitalaria ${ }^{2}$. Además, a este proceso de institucionalización se le suma el progresivo envejecimiento de la población en los países desarrollados, que ha acarreado un incremento de la prevalencia de las enfermedades crónicas y su mortalidad asociada ${ }^{3}$. La gestión del proceso de morir es crucial para las instituciones dedicadas a la salud. Los profesionales son formados en el modelo de una medicina dirigida a la curación ${ }^{4}$, a promover la salud, rehabilitar y prolongar la vida, y quizás por eso el trabajo con personas en el proceso final de la vida es considerado como una de las experiencias más difíciles y estresantes dentro del ejercicio de la profesión ${ }^{5}$.

Los estudiantes de enfermería, a lo largo de sus prácticas clínicas, toman contacto con el sufrimiento del enfermo terminal y su familia. Se ven obligados a enfrentarse tanto a los miedos del paciente como a los suyos propios ${ }^{6}$. Consideran el proceso de morir una de las experiencias más estresantes de las prácticas". La "educación para la muerte" es un ámbito educativo inédito desde nuestra infancia y se trata de forma escasa e irregular en los planes de estudio de los futuros enfermeros ${ }^{8}$. En ocasiones, los estudiantes tienen que proporcionar cuidados postmortem; especialmente lo que más ansiedad les causa es manipular el cuerpo de una persona fallecida, pues, según ellos, carecen de preparación`. Por tanto, si se pudiera incidir en el aspecto emocional de los futuros profesionales de enfermería estaríamos ayudando no solo a estos, sino también al enfermo, ya que la mejor preparación emocional del profesional repercutiría en una mejor calidad de la atención a la persona que se encuentra en la etapa final de su vida ${ }^{10,11}$. Se trata, pues, de que los futuros profesionales utilicen las competencias emocionales para afrontar las situaciones de enfermedad y muerte del paciente. A través del constructo "inteligencia emocional"12 los universitarios deberían ser conscientes de las emociones, comprenderlas y manejarlas, no solo en ellos mismos, sino también en otras personas, utilizando estas para razonar mejor. Hay estudios que manifiestan que los principales estresores para los estudiantes son el sufrimiento ajeno, la comunicación con una persona cercana a la muerte y la propia muerte del paciente ${ }^{13}$. Otros autores destacan que el miedo a la muerte, especialmente la muerte de otros, disminuye significativamente a medida que los alumnos avanzan con su formación ${ }^{14,15}$. Además, los estudiantes que han presenciado una muerte durante las prácticas clínicas presentan menor miedo tanto al propio proceso de morir como al de otro, es decir, la experiencia de la muerte de otra persona ayuda al desarrollo de capacidades y estrategias de afrontamiento eficaces. Estos resultados contrastan con los obtenidos por otros autores ${ }^{16}$, que muestran que los estudiantes de primer curso presentan menor miedo a la muerte, posiblemente porque aún no tienen experiencia clínica alguna.

Durante los últimos años también se ha indagado en el campo de la inteligencia emocional, evaluada a través de las dimensiones "atención", "regulación" y "comprensión o claridad emocional". Así, una investigación concluye que los 
estudiantes con puntuaciones más altas en el área de atención emocional muestran mayor miedo a la muerte, mientras que aquellos con puntuaciones altas en comprensión y regulación de las emociones presentan niveles inferiores de miedo a la muerte ${ }^{15}$. Además, se sugiere la necesidad de formar adecuadamente en competencias emocionales a los futuros profesionales de enfermería para que afronten de una manera más eficaz y bajo un menor coste emocional las situaciones de sufrimiento y muertes. En esta línea, otras investigaciones han reportado resultados idénticos: hay una relación que indica que una mayor comprensión de las emociones y la mayor capacidad de regulación reduce el miedo a la muerte, mientras que una alta atención de los sentimientos lo incrementa ${ }^{17}$. Por último, otros autores sugieren que las enfermeras con niveles más altos en inteligencia emocional afrontan de manera más positiva la muerte, al poseer mejores niveles de adaptación psicológica ${ }^{18}$.

Según la evidencia expuesta y dada la importante relación que parecen tener los aspectos emocionales con la manera de afrontar la muerte por parte de los futuros profesionales de enfermería, el objetivo de este estudio será analizar la relación entre el miedo a la muerte y la inteligencia emocional en los alumnos de cuarto curso del Grado de Enfermería de una universidad pública española, además de describir ambas variables y comparar las diferencias con distintas variables sociodemográficas de la muestra de estudio.

\section{Método}

Es un estudio descriptivo transversal, de diseño comparativo y correlacional.

La población fueron los 164 estudiantes de cuarto curso del Grado de Enfermería de una universidad pública española.

\section{Instrumentos de medida}

Para describir las variables sociodemográficas se utilizó un cuestionario ad hoc de recogida de datos. En este cuestionario también se obtuvieron otros datos sobre el perfil de la muestra (Tabla I), así como la valoración sobre la preparación asistencial y emocional autopercibida para cuidar a enfermos terminales. Mediante esta cuestión se les preguntaba a los estudiantes si se sentían preparados desde un punto de vista asistencial y emocional para cuidar a enfermos terminales. Dicha pregunta podía responderse desde "Nada" (1 punto) a "Mucho" (5 puntos).

La escala de Miedo a la Muerte de Collett-Lester (EMMCL), en su adaptación española ${ }^{19}$, consta de 28 ítems divididos en cuatro subescalas: 1) miedo a la propia muerte; 2) miedo a la muerte de otros; 3 ) miedo al proceso de morir de uno mismo; 4) miedo al proceso de morir de otros. Cada subescala está compuesta por siete ítems con un formato de respuesta de tipo Likert donde las respuestas van desde "No me preocupa nada" (1 punto) hasta "Me preocupa mucho" (5 puntos).

La escala de inteligencia emocional Trait Meta Mood Scale, en la versión reducida en español (TMMS-24) ${ }^{20}$, consta de 24 ítems divididos en tres dimensiones de la inteligencia emocional: 1) atención; 2) claridad; 3) regulación. Cada dimensión está compuesta por ocho ítems con un formato
Tabla I. Características sociodemográficas de los estudiantes de enfermería de una universidad pública española

\begin{tabular}{|c|c|c|}
\hline & & $\mathrm{n}=101(100 \%)$ \\
\hline \multirow{2}{*}{ Género } & Hombres & $26(25,7 \%)$ \\
\hline & Mujeres & $75(74,3 \%)$ \\
\hline \multirow{4}{*}{ Ocupación } & Solo estudian & $51(50,5 \%)$ \\
\hline & $\begin{array}{l}\text { Trabajan de forma } \\
\text { esporádica }\end{array}$ & 27 (26,7 \%) \\
\hline & $\begin{array}{l}\text { Trabajan durante } \\
\text { periodos vacacionales }\end{array}$ & $9(8,9 \%)$ \\
\hline & $\begin{array}{l}\text { Trabajan de forma } \\
\text { continuada }\end{array}$ & $14(13,9 \%)$ \\
\hline \multirow{5}{*}{$\begin{array}{l}\text { Tu primer } \\
\text { contacto con el } \\
\text { hospital }\end{array}$} & Prácticas clínicas & $25(24,8 \%)$ \\
\hline & $\begin{array}{l}\text { Visitas a familiares y } \\
\text { enfermos }\end{array}$ & $49(48,5 \%)$ \\
\hline & $\begin{array}{l}\text { Visitas a amigos } \\
\text { enfermos }\end{array}$ & $3(3 \%)$ \\
\hline & Mi propio ingreso & $22(21,8 \%)$ \\
\hline & Otro & $2(2 \%)$ \\
\hline \multirow{4}{*}{$\begin{array}{l}\text { Acompañamiento } \\
\text { de una persona } \\
\text { durante el } \\
\text { proceso final de } \\
\text { la vida }\end{array}$} & A un paciente & $67(66,3 \%)$ \\
\hline & $\begin{array}{l}\text { A un familiar muy } \\
\text { cercano }\end{array}$ & $7(6,9 \%)$ \\
\hline & A un familiar cercano & $59(58,4 \%)$ \\
\hline & A un amigo & $5(5 \%)$ \\
\hline \multirow{5}{*}{$\begin{array}{l}\text { Acompañamiento } \\
\text { de una persona } \\
\text { durante el } \\
\text { proceso final del } \\
\text { duelo }\end{array}$} & A un amigo & $61(60,4 \%)$ \\
\hline & A un familiar cercano & $59(59,4 \%)$ \\
\hline & Él mismo lo ha vivido & $55(54,4 \%)$ \\
\hline & $\begin{array}{l}\text { A un familiar muy } \\
\text { cercano }\end{array}$ & $55(54,5 \%)$ \\
\hline & $\begin{array}{l}\text { A un familiar del } \\
\text { paciente }\end{array}$ & $52(51,5 \%)$ \\
\hline
\end{tabular}

de respuesta de tipo Likert donde las repuestas van desde "Nada de acuerdo" (1 punto) hasta "Totalmente de acuerdo" (5 puntos).

La recogida de datos se llevó a cabo durante el primer trimestre del curso académico 2015-2016. La administración de los cuestionarios en formato papel tuvo lugar en el aula, durante las prácticas de una asignatura de cuarto curso del Grado en Enfermería. El tiempo empleado fue de un máximo de 30 minutos. Se eligió el último año de la titulación para asegurarse de que los estudiantes hubieran recibido la formación teórica sobre la asistencia final de la vida.

El procesamiento de los datos se realizó utilizando el paquete estadístico SPSS versión 23.0 para Windows. Se utilizó el coeficiente de correlación de Pearson para establecer la relación entre la variable miedo a la muerte y la variable inteligencia emocional, y el coeficiente de correlación de Spearman para estudiar la relación entre el miedo a la muerte y el nivel de preparación asistencial y emocional autopercibido. Por último, se aplicó la prueba T de Student para conocer las diferencias entre hombres y mujeres. 


\section{Consideraciones éticas}

Los cuestionarios fueron acompañados de la hoja de información y del consentimiento verbal y escrito. Se consideró en todo momento el anonimato de los sujetos que formaban parte del estudio, así como el carácter voluntario de su participación.

Durante el periodo del estudio se siguieron las directrices nacionales e internacionales (Código Deontológico y Declaración de Helsinki) y la normativa legal sobre la confidencialidad de los datos (Ley Orgánica 15/1999, de 13 de diciembre, de protección de datos de carácter personal). El estudio fue aprobado por un comité de ética (ULE002-2017), garantizando así el cumplimiento de los aspectos éticos y legales.

\section{Resultados}

Participaron 101 estudiantes (61,6\% de la población), de los que 75 eran mujeres y 26 hombres (Tabla I). La media de edad fue de 22,8 años

Los resultados obtenidos para las variables preparación asistencial y emocional autopercibida para cuidar a enfermos terminales, miedo a la muerte (EMMCL) e inteligencia emocional (TMMS-24) se exponen en la Tabla II.

Se observa que las estudiantes se autoperciben menos preparadas asistencialmente que ellos a la hora de cuidar enfermos terminales $(p=0,01)$. Tal y como se evidencia con la escala EMMCL, las mujeres puntúan significativamente más bajo en "miedo al propio proceso de morir" $(3,61 \pm 0,73)$ y más alto que los hombres en "miedo al proceso de morir de otros" $(3,58 \pm 0,70)$. El género femenino presenta una puntuación significativamente mayor que los hombres para el promedio general de la EMMCL $(3,69 \pm 0,56)$. No se han encontrado diferencias estadísticamente significativas entre hombres y mujeres en el caso de la variable inteligencia emocional.

El análisis de correlación entre el miedo a la muerte y la inteligencia emocional se muestra en la Tabla III. La regulación emocional se correlaciona en negativo de manera signi- ficativa y débil con el "miedo a la muerte propia", "miedo a la muerte de otros" y "miedo al proceso de morir de otros".

No existen diferencias estadísticamente significativas entre el miedo a la muerte y el primer contacto en el hospital, ni con el acompañamiento de una persona en el proceso final de su vida o de su duelo. En la Tabla IV se observa que la preparación asistencial y emocional para cuidar enfermos terminales se correlaciona negativa, leve y significativamente con el miedo a la muerte.

\section{Discusión}

En líneas generales, los resultados obtenidos en la EMMCL son similares a los hallados en otros trabajos realizados en este ámbito ${ }^{14,21,22}$. Aunque todas las subescalas obtuvieron puntuaciones bastante semejantes, la que alcanzó valores más elevados fue el "miedo al propio proceso de morir". Este resultado podría estar relacionado con el hecho de ser personal sanitario y conocer de primera mano la realidad asistencial a través de las prácticas. La muestra estudiada ha estado en contacto con personas en la última fase de su vida, lo que implica un mayor conocimiento de la enfermedad terminal, los síntomas, el sufrimiento y la agonía que padecen algunos pacientes, y de ahí que polaricen más su ansiedad en el proceso de la muerte y no en la muerte como tal.

Además, se confirma la tendencia de la gran mayoría de los estudios previos ${ }^{14,17,19,21}$ y una de las hipótesis de esta investigación: el género femenino presenta puntuaciones significativamente mayores de miedo a la muerte que los hombres. La explicación a este fenómeno, considerado como universal ${ }^{23}$, podría ser que las mujeres poseen mayor facilidad a la hora de expresar y admitir los sentimientos relacionados con la muerte.

Un buen volumen de investigaciones ${ }^{17,20,24}$ relacionadas con la actitud hacia la muerte y el enfermo terminal incluyen la evaluación del nivel de inteligencia emocional a través de la TMMS-24. Resulta casi indispensable conocer cómo canaliza, siente, regula y vive el futuro profesional de enfermería la

Tabla II. Estadística descriptiva y T de Student de la preparación asistencial, emocional, miedo a la muerte (EMMCL), inteligencia emocional (TMMS-24) y el género de los estudiantes de enfermería

\begin{tabular}{llcrrr}
\hline & & Hombres $(\mathbf{n}=\mathbf{2 6})$ & Mujeres $(\mathbf{n}=\mathbf{7 5})$ & Total $(\mathbf{n}=101)$ & $\boldsymbol{p}$ \\
\hline \multirow{2}{*}{ Preparación } & Asistencial & $3,62 \pm 0,90$ & $3,15 \pm 0,88$ & $3,27 \pm 0,90$ & 0,010 \\
\cline { 2 - 5 } & Emocional & $3,38 \pm 1,17$ & $3,08 \pm 0,98$ & $3,16 \pm 1,04$ & 0,122 \\
\hline \multirow{5}{*}{ EMMCL } & Miedo a la propia muerte & $3,15 \pm 1,03$ & $3,52 \pm 0,94$ & $3,42 \pm 0,97$ & 0,156 \\
\cline { 2 - 5 } & Miedo al propio proceso de morir & $3,90 \pm 0,73$ & $3,61 \pm 0,73$ & $4,01 \pm 0,70$ & 0,014 \\
\cline { 2 - 5 } & Miedo a la muerte de otros & $3,57 \pm 0,60$ & $3,37 \pm 0,71$ & $3,64 \pm 0,55$ & 0,31 \\
\cline { 2 - 5 } & Miedo al proceso de morir de otros & $3,25 \pm 0,057$ & $3,58 \pm 0,70$ & $3,5 \pm 0,68$ & 0,018 \\
\cline { 2 - 5 } & Total & $3,35 \pm 0,65$ & $3,69 \pm 0,56$ & $3,6 \pm 0,60$ & 0,020 \\
\hline \multirow{3}{*}{ TMMS-24 } & Atención & $29,2 \pm 6,1$ & $29,1 \pm 6,3$ & $29,1 \pm 6,2$ & 0,93 \\
\cline { 2 - 5 } & Compresión & $27,9 \pm 6,4$ & $28,4 \pm 6,3$ & $28,3 \pm 6,1$ & 0,714 \\
\cline { 2 - 5 } & Regulación & $29,8 \pm 5,5$ & $28,8 \pm 5,6$ & $29,0 \pm 5,5$ & 0,438 \\
\hline
\end{tabular}

EMMCL: escala de Miedo a la Muerte de Collett-Lester. TMMS-24: escala de inteligencia emocional Trait Meta Mood Scale, en la versión reducida en español. 
Tabla III. Correlaciones entre miedo a la muerte (EMMCL) e inteligencia emocional (TMMS-24) de los estudiantes de enfermería

\begin{tabular}{|c|c|c|c|c|c|}
\hline & & & \multicolumn{3}{|c|}{ TMMS-24 } \\
\hline & & & Atención & Compresión & Regulación \\
\hline \multirow{10}{*}{ EMMCL } & \multirow{2}{*}{ Miedo a la muerte propia } & Pearson & 0,138 & 0,021 & $-0,201$ \\
\hline & & Sig. & 0,167 & 0,835 & $0,043^{*}$ \\
\hline & \multirow{2}{*}{ Miedo al propio proceso de morir } & Pearson & 0,136 & 0,024 & $-0,069$ \\
\hline & & Sig. & 0,177 & 0,813 & 0,490 \\
\hline & \multirow{2}{*}{ Miedo a la muerte de otros } & Pearson & 0,118 & $-0,153$ & $-0,226$ \\
\hline & & Sig. & 0,238 & 0,128 & $0,023^{*}$ \\
\hline & \multirow{2}{*}{ Miedo al proceso de otros } & Pearson & 0,046 & $-0,167$ & $-0,199$ \\
\hline & & Sig. & 0,647 & 0,096 & $0,046^{*}$ \\
\hline & \multirow{2}{*}{ Total miedo a la muerte } & Pearson & 0,140 & $-0,070$ & $-0,216$ \\
\hline & & Sig. & 0,162 & 0,486 & $0,030^{*}$ \\
\hline
\end{tabular}

*La correlación es significativa en el nivel 0,01.

EMMCL: escala de Miedo a la Muerte de Collett-Lester. TMMS-24: escala de inteligencia emocional Trait Meta Mood Scale, en la versión reducida en español.

Tabla IV. Correlaciones entre autopercepción emocional y asistencial y miedo a la muerte (EMMCL) de los estudiantes de enfermería

\begin{tabular}{cccc}
\hline & $\begin{array}{c}\text { Autopercepción } \\
\text { preparación } \\
\text { asistencial }\end{array}$ & $\begin{array}{c}\text { Autopercepción } \\
\text { preparación } \\
\text { emocional }\end{array}$ \\
\hline \multirow{2}{*}{ EMMCL } & Spearman & $-0,328$ & $-0,323$ \\
\cline { 2 - 4 } & Sig. & $0,001^{*}$ & $0,001^{*}$ \\
\hline
\end{tabular}

*La correlación es significativa en el nivel 0,01.

EMMCL: escala de Miedo a la Muerte de Collett-Lester.

red de emociones que rigen el proceso de morir. Se evidenció que las puntuaciones mayores en la regulación de las emociones se asocian con valores más bajos en el miedo a la muerte. En particular, el área de regulación se relaciona de la misma manera con tres de las cuatro subescalas de la EMMCL: "miedo a la muerte propia", "miedo a la muerte de otros" y "miedo al proceso de morir de otros". De este modo, los hallazgos de este estudio son parecidos para el área de regulación emocional a los resultados obtenidos en anteriores investigaciones, que demuestran que la inteligencia emocional está íntimamente relacionada con el nivel de miedo a la muerte, al encontrar correlaciones estadísticamente significativas para dos de las tres áreas de la inteligencia emocional ${ }^{17} \mathrm{o}$ incluso para las tres áreas ${ }^{11,21,24}$. Los autores de estos trabajos exponen que los resultados obtenidos se deben a que las personas con niveles óptimos de adaptación psicológica muestran puntuaciones moderadas o bajas en el área de atención emocional y niveles altos de comprensión y regulación de las emociones.

Los resultados de la presente investigación se deben interpretar con prudencia por las limitaciones que presenta el estudio. El diseño comparativo correlacional no puede esta- blecer relaciones de causalidad. Sería interesante plantear estudios longitudinales a largo plazo. Además, el pequeño tamaño, la falta de homogeneidad para la variable género y la titulación de la muestra indican dificultad para extrapolar los datos a la población de estudiantes universitarios.

\section{Conclusiones}

La puntuación media alcanzada para las variables miedo a la muerte e inteligencia emocional en la población de estudio presenta niveles parecidos a poblaciones semejantes. Además, la escala que alcanzó valores más elevados fue el "miedo al propio proceso de morir". Es decir, parece que no es tanto la idea propia de la muerte lo que despierta mayor temor entre los alumnos de enfermería, sino la muerte como proceso: el miedo al dolor, la degeneración física y mental, la pérdida de facultades, la incertidumbre sobre cómo se afrontará, etc.

Las estudiantes mujeres se autoperciben menos preparadas asistencialmente que los hombres a la hora de cuidar enfermos terminales. Además, ellas se muestran con mayores niveles de miedo a la muerte que los hombres.

Se ha demostrado la relación entre la inteligencia emocional y el miedo a la muerte; así, la capacidad de regular las emociones en los estudiantes de cuarto curso del Grado de Enfermería se relaciona con un menor miedo a la muerte.

La formación del estudiante en el ámbito de los cuidados al final de la vida debe incluir estrategias que favorezcan el desarrollo de competencias socioemocionales para así dotar a los futuros enfermeros de recursos eficaces a la hora de cuidar y cuidarse eficazmente.

\section{Conflicto de intereses}

Ninguno. 


\section{Financiación}

El presente trabajo ha sido financiado por la Universidad de León (España) dentro del Plan de Apoyo a los Grupos de Innovación Docente (PAGID) titulado "El Juego como herramienta para comprender y ayudar en el Duelo".

\section{Bibliografía}

1. Cruz-Quintana F, García-Caro M. SOS. Dejadme morir. Ayudando a aceptar la muerte. 1. ${ }^{\mathrm{a}}$ ed. Madrid: Pirámide; 2007.

2. Edo-Gual M. Actitudes ante la muerte y factores relacionados de los estudiantes de enfermería en la comunidad autónoma de Catalunya [Internet] [Tesis]. Universitat Internacional de Catalunya, Facultad de Medicina y Ciencias de la Salud; 2015 [citado el 21 de agosto de 2017]. Disponible en: http://www.tdx.cat/ handle/10803/317380.

3. Gómez-Batiste X, Espinosa J, Porta-Sales J, Benito E. Modelos de atención, organización y mejora de la calidad para la atención de los enfermos en fase terminal y su familia: aportación de los cuidados paliativos. Med Clin. 2010;135:83-9.

4. Benítez-del-Rosario M, Salinas-Martín A. Cuidados paliativos y atención primaria: aspectos de organización. $1{ }^{\mathrm{a}}{ }^{\mathrm{e}}$ ed. Barcelona: Springer-Verlag Ibérica; 2000.

5. Costello J. Dying well: nurses' experiences of 'good and bad' deaths in hospital. J Adv Nurs. 2006;54:594-601.

6. Tomás-Sábado J, Gómez-Benito J. Variables relacionadas con la ansiedad ante la muerte. Rev Psicol Gen y Apl Rev la Fed Española Asoc Psicol. 2003;56:257-79.

7. Chocrón S, Alfaya MM, Villaverde C, Roa JM, Benbunan B, Cruz F. El impacto emotivo del hospital: implicaciones en la formación universitaria de los estudiantes de enfermería. Rev ROL enfermería. 2005;28:35-42.

8. Hanzeliková-Pogrányivá A, García-López M, Conty Serrano M, López-Dávila-Sánchez B, Barriga-Martín J, Martín-Conty J. Reflexiones de los alumnos de Enfermería sobre el proceso de la muerte. Enferm Glob. 2014;33:133-44.

9. Parry M. Student nurses' experience of their first death in clinical practice. Int J Palliat Nurs. 2011;17:448-53.

10. Edo-Gual M, Monforte-Royo C, Aradilla-Herrero A, Tomás-Sábado J. Death attitudes and positive coping in Spanish nursing undergraduates: a cross-sectional and correlational study. J Clin Nurs. 2015;24:2429-38.

11. Bermejo JC, Villacieros M, Hassoun H. Actitudes hacia el cuidado de pacientes al final de la vida y miedo a la muerte en una muestra de estudiantes sociosanitarios. Med Paliat. 2018;25:168-74.
12. López-Fernández C. Inteligencia emocional y relaciones interpersonales en los estudiantes de enfermería. Educ Médica. 2015; 16:83-92.

13. Burnard P, Edwards D, Bennett K, Thaibah H, Tothova V, Baldacchino $D$, et al. A comparative, longitudinal study of stress in student nurses in five countries: Albania, Brunei, the Czech Republic, Malta and Wales. Nurse Educ Today. 2008;28:134-45.

14. Edo-Gual M, Tomás-Sábado J, Aradilla-Herrero A. Miedo a la muerte en estudiantes de enfermería. Enfermería Clínica. 2011;21:129-35.

15. Aradilla-Herrero A, Tomas-Sabado J, Gómez-Benito J. Death attitudes and emotional intelligence in nursing students. OMEGA-J Death Dying. 2013;66:39-55.

16. Mondragón-Sánchez EJ, Torre Cordero EA, Morales Espinoza M de $L$, Landeros-Olvera EA. A comparison of the level of fear of death among students and nursing professionals in Mexico. Rev Lat Am Enfermagem. 2015;23:323-8.

17. Espinoza-Venegas M, Sanhueza O. Miedo a la muerte y su relación con la inteligencia emocional de estudiantes de enfermería de Concepción. Acta Paul Enferm. 2012;25:607-13.

18. Hopkinson JB, Hallett CE, Luker KA. Everyday death: How do nurses cope with caring for dying people in hospital? Int J Nurs Stud. 2005;42:125-33.

19. Tomás-Sábado J, Limonero JT, Abdel-Khalek AM. Spanish adaptation of the Collett-Lester fear of death scale. Death Stud. 2007;31:249-60.

20. Fernandez-Berrocal P, Extremera N, Ramos N. Validity and reliability of the Spanish modified version of the Trait Meta-Mood Scale. Psychol Rep. 2004;94:751-5.

21. Colell-Brunet $M$. Análisis de las actitudes ante la muerte y el enfermo al final de la vida en estudiantes de enfermería de Andalucía y Cataluña [Internet] [Tesis]. Universitat Autónoma de Barcelona, Departament de Psicología Básica, Evolutiva i de I'Educació; 2005 [citado 4 Nov 2015]. Disponible en: http:// ddd.uab.cat/record/38526.

22. López-Alonso Al, Fernández-Martínez ME, Liébana-Presa C, Vázquez-Casares AM, Castro González MP. Los juegos experimentales de aula: una herramienta didáctica en cuidados paliativos. Rev Esc Enferm USP. 2018;52.

23. Pollack JM. Correlates of death anxiety: A review of empirical studies. Omega. 1979;10:97-121.

24. González-Fernández S. Actitudes ante la muerte en estudiantes de grado de enfermería [Internet] [Tesis]. Universidad Complutense de Madrid, Facultad de Enfermería, Fisioterapia y Podología; 2015 [citado 21 Ago 2017]. Disponible en: http:// eprints.ucm.es/34366/1/T36685.pdf. 